\title{
On the sum of contractive type of mappings II: maps on different classes
}

Edixon M Rojas ${ }^{1 *}$ and José R Morales²

"Correspondence: edixonr@gmail.com

${ }^{1}$ Departamento de Matemáticas, Pontificia Universidad Javeriana, Bogotá, Colombia Full list of author information is available at the end of the article

\begin{abstract}
In this paper we will show that the sum of two mappings belonging to different contractive classes gives one map in another class that should be different from the two classes of mappings initially considered. As a consequence of this, the existence of a fixed point for the mapping result of the sum of two contractive type of mapping can be guaranteed.
\end{abstract}

MSC: Primary 47H09; 47H10; secondary 54E50

Keywords: contractive mapping; fixed point; Banach space

\section{Preliminaries}

In [1] were given the conditions under which the function resulting from the sum of two maps belonging to a contractive class of mappings satisfies the same kind of contraction inequality. Here, for a mapping $T=R+S$, where $R$ and $S$ satisfy different contractive inequalities, we are going to investigate conditions to assure that $T$ be a contractive type mapping.

In order to attain our aim, we will use a reverse triangle inequality given by Diaz and Metcalf in [2].

Proposition 1.1 (Diaz-Metcalf, 1966, [2]) If $F: X \longrightarrow \mathbb{R}$ is a linear functional of a unit norm defined on the normed linear space $X$ endowed with the norm $\|\cdot\|$ and the vectors $x_{1}, \ldots, x_{n}$ satisfy the condition

$$
0 \leq r \leq F\left(x_{i}\right), \quad i \in\{1, \ldots, n\}
$$

then

$$
r \sum_{i=1}^{n}\left\|x_{i}\right\| \leq\left\|\sum_{i=1}^{n} x_{i}\right\|
$$

where equality holds if and only if both

$$
F\left(\sum_{i=1}^{n} x_{i}\right)=r \sum_{i=1}^{n}\left\|x_{i}\right\|
$$

@2014 Rojas and Morales; licensee Springer. This is an Open Access article distributed under the terms of the Creative Commons Attribution License (http://creativecommons.org/licenses/by/2.0), which permits unrestricted use, distribution, and reproduction in any medium, provided the original work is properly cited. 
and

$$
F\left(\sum_{i=1}^{n} x_{i}\right)=\left\|\sum_{i=1}^{n} x_{i}\right\| .
$$

In this paper we are going to consider mappings satisfying the next classical result.

Theorem 1.2 Let $(M, d)$ be a complete metric space and $T: M \longrightarrow M$ a map. Then $T$ has a fixed point in $M$ if it satisfies any of the following conditions:

$B C(\alpha)$ (Banach, 1922; see [3]) $T$ is an $\alpha$-contraction or Banach contraction, that is,

$$
d(T x, T y) \leq \alpha d(x, y) \quad \forall x, y \in M, 0 \leq \alpha<1
$$

$K A(\alpha)$ (Kannan, 1969, 1971, [4, 5]) T satisfies: there is $\alpha \in\left[0, \frac{1}{2}\right)$ such that

$$
d(T x, T y) \leq \alpha(d(x, T x)+d(y, T y)) \quad \forall x, y \in M
$$

$C H(\alpha)$ (Chatterjea, 1972, [6]) T satisfies the following condition: there is $\alpha \in\left[0, \frac{1}{2}\right)$ such that

$$
d(T x, T y) \leq \alpha(d(x, T y)+d(y, T x)) \quad \forall x, y \in M
$$

$R E\left(a_{1}, a_{2}, a_{3}\right)$ (Reich, 1971, [7-9]) T satisfies

$$
d(T x, T y) \leq a_{1} d(x, y)+a_{2} d(x, T x)+a_{3} d(y, T y)
$$

for all $x, y \in M$, with $0 \leq a_{1}+a_{2}+a_{3}<1$.

$R H\left(a_{1}, a_{2}, a_{3}\right)$ (Rhoades, 1977, [10] or see [11]) T satisfies

$$
d(T x, T y) \leq a_{1} d(x, y)+a_{2} d(x, T y)+a_{3} d(y, T x)
$$

for all $x, y \in M, 0 \leq a_{1}+a_{2}+a_{3}<1$.

$H R\left(a_{1}, a_{2}, a_{3}, a_{4}, a_{5}\right)$ (Hardy-Rogers, 1973, [12] or see $[13,14]$ for instance) $\forall x, y \in M, T$ satisfies: there are $a_{i} \geq 0$ such that $A=\sum_{i=1}^{5} a_{i}<1$ and

$$
d(T x, T y) \leq a_{1} d(x, y)+a_{2} d(x, T x)+a_{3} d(y, T y)+a_{4} d(x, T y)+a_{5} d(y, T x) .
$$

$D(a, b)$ (Nova, 1986, [15] or see [16, 17] for instance) $K \subset M$ closed and $T: K \longrightarrow K$ an arbitrary operator that satisfies the following condition, for $a, b \geq 0$ and any $x, y \in K$ :

$$
d(T x, T y) \leq a d(x, y)+b[d(x, T x)+d(y, T y)] .
$$

We shall say that $T$ belongs to or is of class $B C(\alpha)$ (respectively, $K A(\alpha), C H(\alpha)$, $\left.R E\left(a_{1}, a_{2}, a_{3}\right), R H\left(a_{1}, a_{2}, a_{3}\right), H R\left(a_{1}, a_{2}, a_{3}, a_{4}, a_{5}\right), D(a, b)\right)$ when $T$ satisfies the condition $B C(\alpha)$ (respectively, $K A(\alpha), C H(\alpha), R E\left(a_{1}, a_{2}, a_{3}\right), R H\left(a_{1}, a_{2}, a_{3}\right), H R\left(a_{1}, a_{2}, a_{3}, a_{4}, a_{5}\right)$, $D(a, b))$ where $\alpha$ indicates the contraction's constant (the same indicates the parameters in each of the remaining classes). 
The conditions above are in general independent of each other. Examples showing that can be found in [18]. A comparison of these and other classes of contractive type of mappings is given by Rhoades in [10].

\section{The sum of two mappings belonging to different classes of contractive type of maps}

In this section we will study the sum of two mappings belonging to the different classes of contractive maps considered in Theorem 1.2. Our principal objective is the existence of a fixed point for the map resulting from the sum of two contractive type of mappings. For this reason, we are going to consider the contractive parameters to be sufficiently small such that the uniqueness of the fixed point can be guaranteed for the mappings on each class (Theorem 1.2).

Let $(X,\|\cdot\|)$ be a Banach space and $T, S: X \longrightarrow X$ be two mappings. To establish our results we are going to assume that the Diaz-Metcalf's Theorem is satisfies for $(I-T) x$ and $(I-S) x$ for each $x \in X$; i.e.,

$$
\left\{\begin{array}{l}
0<r \leq F(x-T x), \\
0<r \leq F(x-S x),
\end{array} \quad \text { for all } x \in X .\right.
$$

We would like to point out that the case when $0=r=F(T x-x)=F(S x-x)$ corresponds to the case when $x$ in the common fixed point for the pair $(T, S)$ which is unique or does not exist. This fact justifies that in our results we consider only the case $r>0$.

Theorem 2.1 Let $X$ be a Banach space, and $T, S: B_{X}(r) \longrightarrow B_{X}(r)$. Assume the following conditions hold:

(a) $T \in B C(\alpha)$.

(b) For each $x \in X$, the Diaz-Metcalf's condition (1) holds for $r>0$.

Then:

(i) $T+S \in D(\alpha, \beta / r)$, if $S \in K A(\beta)$.

(ii) $T+S \in R H(\alpha, \beta / r, \beta / r)$, if $S \in C H(\beta)$.

(iii) $T+S \in R E\left(a, a_{2} / r, a_{3} / r\right)$, if $S \in R E\left(a_{1}, a_{2}, a_{3}\right), a=\alpha+a_{1}$.

(iv) $T+S \in R H\left(a, a_{2} / r, a_{3} / r\right)$, if $S \in R H\left(a_{1}, a_{2}, a_{3}\right), a=\alpha+a_{1}$.

(v) $T+S \in H R\left(a, a_{2} / r, a_{3} / r, a_{4} / r, a_{5} / r\right)$, if $S \in H R\left(a_{1}, a_{2}, a_{3}, a_{4}, a_{5}\right), a=\alpha+a_{1}$.

(vi) $T+S \in D(\mu, b / r)$, if $S \in D(a, b), \mu=\alpha+a$.

Proof We are going to prove only statement (vi), the remaining proofs are similar.

Let $x, y \in B_{X}(r)$,

$$
\begin{aligned}
& \|T x-T y\| \leq \alpha\|x-y\|, \\
& \|S x-S y\| \leq a\|x-y\|+b[\|x-S x\|+\|y-S y\|] ;
\end{aligned}
$$

then

$$
\|T x-T y\|+\|S x-S y\| \leq \alpha\|x-y\|+a\|x-y\|+b[\|x-S x\|+\|y-S y\|]
$$


now, adding the term $b[\|x-T x\|+\|y-T y\|]$, to the right hand side of the above inequality, we have

$$
\begin{aligned}
\|(T+S) x-(T+S) y\| \leq & (\alpha+a)\|x-y\|+b[\|x-S x\|+\|y-S y\|] \\
& +b[\|x-T x\|+\|y-T y\|] \\
= & \mu\|x-y\|+b[\|x-S x\|+\|y-S y\| \\
& +\|x-T x\|+\|y-T y\|] \quad \text { where } \mu=\alpha+a .
\end{aligned}
$$

From condition (b) we get the following:

$$
\begin{aligned}
\|(T+S) x-(T+S) y\| \leq & \mu\|x-y\|+\frac{b}{r}[\|2 x-(T+S) x\|+\|2 y-(T+S) y\|] \\
\leq & \mu\|x-y\|+\frac{b}{r}(\|x\|+\|y\|)+b[\|x-(T+S) x\| \\
& +\|y-(T+S) y\|] \\
\leq & \mu\|x-y\|+\frac{b}{r}[\|x-(T+S) x\| \\
& +\|y-(T+S) y\|]+2 b .
\end{aligned}
$$

We choose $b$ sufficiently small such that the following inequality is preserved:

$$
\|(T+S) x-(T+S) y\| \leq \mu\|x-y\|+\frac{b}{r}[\|x-(T+S) x\|+\|y-(T+S) y\|]
$$

i.e. $T+S \in D\left(\mu, \frac{b}{r}\right)$.

The proof of the five statements remaining follows in a similar way. Add a convenient term; it may depend on the parameters for the corresponding class, in the case that it is necessary. For example, for the proof of (ii) we need to add the following term:

$$
\beta[\|x-T x\|+\|y-T y\|]
$$

Remark 1 We would like to point out that the values of the contractive parameters are not unique. For instance, if we assume

$$
b \leq \inf _{x, y \in B_{X}(r)}\left\{\frac{1}{2 \beta r}[\|x-(T+S) x\|+\|y-(T+S) y\|]\right\},
$$

where $\beta>0$, then from the inequality (2), the inequality (3) holds for $\frac{1}{r}\left(b+\frac{1}{\beta}\right)$ instead of $\frac{b}{r}$. This means that $T+S \in D\left(\mu, \frac{1}{r}\left(b+\frac{1}{\beta}\right)\right)$.

In a similar way, different assumptions on the contractive parameters of each one of the classes of mappings in consideration here give different values for the contractive parameters on the resulting class.

Theorem 2.2 Let $X$ be a Banach space, and $T, S: B_{X}(r) \longrightarrow B_{X}(r)$. Assume the following conditions hold:

(a) $T \in K A(\alpha)$. 
(b) For each $x \in X$, the Diaz-Metcalf's condition (1) holds.

Then:

(i) $T+S \in H R(0, \alpha / r, \alpha / r, \beta / r, \beta / r)$, if $S \in C H(\beta)$.

(ii) $T+S \in R E\left(a_{1}, a / r, b / r\right)$, if $S \in R E\left(a_{1}, a_{2}, a_{3}\right), a=\max \left(\alpha, a_{2}\right), b=\max \left(\alpha, a_{3}\right)$.

(iii) $T+S \in H R\left(a_{1}, \alpha / r, \alpha / r, a_{2} / r, a_{3} / r\right)$, if $S \in R H\left(a_{1}, a_{2}, a_{3}\right)$.

(iv) $T+S \in H R\left(a_{1}, a / r, b / r, a_{4} / r, a_{5} / r\right)$, if $S \in H R\left(a_{1}, a_{2}, a_{3}, a_{4}, a_{5}\right), a=\max \left(\alpha, a_{2}\right)$, $b=\max \left(\alpha, a_{3}\right)$.

(v) $T+S \in D(a, \mu / r)$, if $S \in D(a, b), \mu=\max (\alpha, b)$.

Proof In this case we are going to prove only statement (iv), the other proofs are analogous, adding the corresponding term (in the case when it is necessary).

Let $x, y \in B_{X}(r)$,

$$
\begin{aligned}
& \|T x-T y\| \leq \alpha[\|x-T x\|+\|y-T y\|], \\
& \|S x-S y\| \leq a_{1}\|x-y\|+a_{2}\|x-S x\|+a_{3}\|y-S y\|+a_{4}\|x-S y\|+a_{5}\|y-S x\| ;
\end{aligned}
$$

then

$$
\begin{aligned}
&\|T x-T y\|+\|S x-S y\| \\
& \leq \alpha\|x-T x\|+\alpha\|y-T y\|+a_{1}\|x-y\|+a_{2}\|x-S x\| \\
&+a_{3}\|y-S y\|+a_{4}\|x-S y\|+a_{5}\|y-S x\| \\
& \leq a_{1}\|x-y\|+a(\|x-T x\|+\|x-S x\|)+b(\|y-T y\|+\|y-S y\|) \\
&+a_{4}\|x-S y\|+a_{5}\|y-S x\| \quad \text { where } a=\max \left(\alpha, a_{2}\right), b=\max \left(\alpha, a_{3}\right) .
\end{aligned}
$$

Now, adding $a_{4}\|x-T y\|+a_{5}\|y-T x\|$ to the right hand side of the above inequality, we have

$$
\begin{aligned}
\|(T+S) x-(T+S) y\| \leq & a_{1}\|x-y\|+a[\|x-T x\|+\|x-S x\|] \\
& +b[\|y-T y\|+\|y-S y\|] \\
& +a_{4}[\|x-S y\|+\|x-T y\|] \\
& +a_{5}[\|y-S x\|+\|y-T x\|] .
\end{aligned}
$$

Condition (b) implies

$$
\begin{aligned}
&\|(T+S) x-(T+S) y\| \\
& \leq a_{1}\|x-y\|+\frac{a}{r}\|2 x-(T+S) x\|+\frac{b}{r}\|2 y-(T+S) y\| \\
&+\frac{a_{4}}{r}\|2 x-(T+S) y\|+\frac{a_{5}}{r}\|2 y-(T+S) x\| \\
& \leq a_{1}\|x-y\|+\frac{a}{r}\|x-(T+S) x\|+\frac{b}{r}\|y-(T+S) y\| \\
&+\frac{a_{4}}{r}\|x-(T+S) y\|+\frac{a_{5}}{r}\|y-(T+S) x\|+a+b+a_{4}+a_{5} .
\end{aligned}
$$


Again, using the same reasoning as for the results before, we conclude that

$$
\begin{aligned}
\|(T+S) x-(T+S) y\| \leq & a_{1}\|x-y\|+\frac{a}{r}\|x-(T+S) x\|+\frac{b}{r}\|y-(T+S) y\| \\
& +\frac{a_{4}}{r}\|x-(T+S) y\|+\frac{a_{5}}{r}\|y-(T+S) x\|,
\end{aligned}
$$

hence $T+S \in H R\left(a_{1}, a / r, b / r, a_{4} / r, a_{5} / r\right), a=\max \left(\alpha, a_{2}\right), b=\max \left(\alpha, a_{3}\right)$.

Theorem 2.3 Let $X$ be a Banach space, and $T, S: B_{X}(r) \longrightarrow B_{X}(r)$. Assume the following conditions hold:

(a) $T \in C H(\alpha)$.

(b) For each $x \in X$, the Diaz-Metcalf's condition (1) holds.

Then:

(i) $T+S \in H R\left(a_{1}, \alpha / r, \alpha / r, a_{2} / r, a_{3} / r\right)$, if $S \in R E\left(a_{1}, a_{2}, a_{3}\right)$.

(ii) $T+S \in R H\left(a_{1}, a / r, b / r\right)$, if $S \in R H\left(a_{1}, a_{2}, a_{3}\right), a=\max \left(\alpha, a_{2}\right), b=\max \left(\alpha, a_{3}\right)$.

(iii) $T+S \in H R\left(a_{1}, a_{2} / r, a_{3} / r, a / r, b / r\right)$, if $S \in H R\left(a_{1}, a_{2}, a_{3}, a_{4}, a_{5}\right), a=\max \left(\alpha, a_{4}\right)$, $b=\max \left(\alpha, a_{5}\right)$.

(iv) $T+S \in H R(a, b / r, b / r, \alpha / r, \alpha / r)$, if $S \in D(a, b)$.

Proof We prove (i). Let $x, y \in B_{X}(r)$,

$$
\begin{aligned}
& \|T x-T y\| \leq \alpha[\|x-T y\|+\|y-T x\|], \\
& \|S x-S y\| \leq a_{1}\|x-y\|+a_{2}\|x-S x\|+a_{3}\|y-S y\| .
\end{aligned}
$$

Then

$$
\begin{gathered}
\|T x-T y\|+\|S x-S y\| \leq \\
\alpha\|x-T y\|+\alpha\|y-T x\|+a_{1}\|x-y\| \\
+a_{2}\|x-S x\|+a_{3}\|y-S y\| .
\end{gathered}
$$

Now, adding the following term to the right hand side of the inequality above:

$$
\alpha\|x-S y\|+\alpha\|y-S x\|+a_{2}\|x-T x\|+a_{3}\|y-T y\|,
$$

we have

$$
\begin{aligned}
\|(T+S) x-(T+S) y\| \leq & a_{1}\|x-y\|+\alpha[\|x-T y\|+\|x-S y\|] \\
& +\alpha[\|y-T x\|+\|y-S x\|] \\
& +a_{2}[\|x-T x\|+\|x-S x\|] \\
& +a_{3}[\|y-S y\|+\|y-T y\|] .
\end{aligned}
$$

Using the Diaz-Metcalf inequality we get

$$
\begin{aligned}
& \|(T+S) x-(T+S) y\| \\
& \quad \leq a_{1}\|x-y\|+\frac{\alpha}{r}\|2 x-(T+S) y\|+\frac{\alpha}{r}\|2 y-(T+S) x\|
\end{aligned}
$$




$$
\begin{aligned}
& +\frac{a_{2}}{r}\|2 x-(T+S) x\|+\frac{a_{3}}{r}\|2 y-(T+S) y\| \\
\leq & a_{1}\|x-y\|+\frac{\alpha}{r}\|x-(T+S) y\|+\frac{\alpha}{r}\|y-(T+S) x\| \\
& +\frac{a_{2}}{r}\|x-(T+S) x\|+\frac{a_{3}}{r}\|y-(T+S) y\|+2 \alpha+a_{2}+a_{3} .
\end{aligned}
$$

Since $2 \alpha+a_{2}+a_{3}$ can be as small as we please we have

$$
\begin{aligned}
\|(T+S) x-(T+S) y\| \leq & a_{1}\|x-y\|+\frac{\alpha}{r}\|x-(T+S) y\|+\frac{\alpha}{r}\|y-(T+S) x\| \\
& +\frac{a_{2}}{r}\|x-(T+S) x\|+\frac{a_{3}}{r}\|y-(T+S) y\|
\end{aligned}
$$

i.e. $T+S \in H R\left(a_{1}, \alpha / r, \alpha / r, a_{2} / r, a_{3} / r\right)$.

The remaining statements follow as above.

Theorem 2.4 Let $X$ be a Banach space, and $T, S: B_{X}(r) \longrightarrow B_{X}(r)$. Assume the following conditions hold:

(a) $T \in R E\left(a_{1}, a_{2}, a_{3}\right)$.

(b) For each $x \in X$, the Diaz-Metcalf's condition (1) holds.

Then:

(i) $T+S \in H R\left(a, a_{2} / r, a_{3} / r, b_{2} / r, b_{3} / r\right)$, if $S \in R H\left(b_{1}, b_{2}, b_{3}\right), a=a_{1}+b_{1}$.

(ii) $T+S \in H R\left(a, b / r, c / r, b_{4} / r, b_{5} / r\right)$, if $S \in H R\left(b_{1}, b_{2}, b_{3}, b_{4}, b_{5}\right), a=a_{1}+b_{1}$, $b=\max \left(a_{2}, b_{2}\right), c=\max \left(a_{3}, b_{3}\right)$.

(iii) $T+S \in D(\alpha, \mu / r)$, if $S \in D(a, b), \alpha=a_{1}+a, \mu=\max \left(a_{2}, a_{3}, b\right)$.

Proof We now consider (ii). Let $x, y \in B_{X}(r)$,

$$
\begin{aligned}
\|T x-T y\| \leq & a_{1}\|x-y\|+a_{2}\|x-T x\|+a_{3}\|y-T y\|, \\
\|S x-S y\| \leq & b_{1}\|x-y\|+b_{2}\|x-S x\|+b_{3}\|y-S y\| \\
& +b_{4}\|x-S y\|+b_{5}\|y-S x\|
\end{aligned}
$$

then

$$
\begin{aligned}
& \|T x-T y\|+\|S x-S y\| \\
& \leq \quad\left(a_{1}+b_{1}\right)\|x-y\|+a_{2}\|x-T x\|+b_{2}\|x-S x\| \\
& \quad+a_{3}\|y-T y\|+b_{3}\|y-S y\|+b_{4}\|x-S y\|+b_{5}\|y-S x\| \\
& \leq a\|x-y\|+b(\|x-T x\|+\|x-S x\|) \\
& \quad+c(\|y-T y\|+\|y-S y\|)+b_{4}\|x-S y\|+b_{5}\|y-S x\| \\
& \quad \text { where } a=a_{1}+b_{1}, b=\max \left(a_{2}, b_{2}\right), c=\max \left(a_{3}, b_{3}\right) .
\end{aligned}
$$

Now, adding the following term to the right hand side of the inequality above:

$$
b_{4}\|x-T y\|+b_{5}\|y-T x\|,
$$


we have

$$
\begin{aligned}
\| T x- & T y\|+\| S x-S y \| \\
\leq & a\|x-y\|+b(\|x-T x\|+\|x-S x\|) \\
& +c(\|y-T y\|+\|y-S y\|)+b_{4}(\|x-S y\|+\|x-T y\|) \\
& +b_{5}(\|y-S x\|+\|y-T x\|) .
\end{aligned}
$$

Using the Diaz-Metcalf condition we obtain

$$
\begin{aligned}
&\|T x-T y\|+\|S x-S y\| \\
& \leq a\|x-y\|+\frac{b}{r}\|2 x-(T+S) x\|+\frac{c}{r}\|2 y-(T+S) y\| \\
&+\frac{b_{4}}{r}\|2 x-(T+S) y\|+\frac{b_{5}}{r}\|2 y-(T+S) x\| \\
& \leq a\|x-y\|+\frac{b}{r}\|x-(T+S) x\|+\frac{c}{r}\|y-(T+S) y\| \\
&+\frac{b_{4}}{r}\|x-(T+S) y\|+\frac{b_{5}}{r}\|y-(T+S) x\|+b+c+b_{4}+b_{5} .
\end{aligned}
$$

By reasoning as above, we conclude that

$$
\begin{aligned}
\|(T+S) x-(T+S) y\| \leq & a\|x-y\|+\frac{b}{r}\|x-(T+S) x\|+\frac{c}{r}\|y-(T+S) y\| \\
& +\frac{b_{4}}{r}\|x-(T+S) y\|+\frac{b_{5}}{r}\|y-(T+S) x\| .
\end{aligned}
$$

Thus $T+S \in H R\left(a, b / r, c / r, b_{4} / r, b_{5} / r\right), a=a_{1}+b, b=\max \left(a_{2}, b\right), c=\max \left(a_{3}, b_{3}\right)$.

Theorem 2.5 Let $X$ be a Banach space, and $T, S: B_{X}(r) \longrightarrow B_{X}(r)$. Assume the following conditions hold:

(a) $T \in R H\left(a_{1}, a_{2}, a_{3}\right)$.

(b) For each $x \in X$, the Diaz-Metcalf's condition (1) holds.

Then:

(i) $T+S \in H R\left(a, b_{2} / r, b_{3} / r, b / r, c / r\right)$, if $S \in H R\left(b_{1}, b_{2}, b_{3}, b_{4}, b_{5}\right), a=a_{1}+b_{1}$, $b=\max \left(a_{2}, b_{4}\right), c=\max \left(a_{3}, b_{5}\right)$.

(ii) $T+S \in H R\left(a, \mu / r, \mu / r, a_{2} / r, a_{3} / r\right)$, if $S \in D(\alpha, \mu), a=a_{1}+\alpha$.

Proof We treat (ii). Let $x, y \in B_{X}(r)$,

$$
\begin{aligned}
& \|T x-T y\| \leq a_{1}\|x-y\|+a_{2}\|x-T y\|+a_{3}\|y-T x\|, \\
& \|S x-S y\| \leq \alpha\|x-y\|+\mu[\|x-S x\|+\|y-S y\|] ;
\end{aligned}
$$

then

$$
\begin{aligned}
\|T x-T y\|+\|S x-S y\| \leq & \left(a_{1}+\alpha\right)\|x-y\|+\mu\|x-S x\|+\mu\|y-S y\| \\
& +a_{2}\|x-T y\|+a_{3}\|y-T x\| .
\end{aligned}
$$


Now, adding the following term to the right hand side of the inequality above:

$$
\mu\|x-T y\|+\mu\|y-T y\|+a_{2}\|x-S y\|+a_{3}\|y-S x\|,
$$

we have

$$
\begin{aligned}
\| T x- & T y\|+\| S x-S y \| \\
\leq & \left(a_{1}+\alpha\right)\|x-y\|+\mu[\|x-T x\|+\|x-S x\|] \\
& +\mu[\|y-T y\|+\|y-S y\|]+a_{2}[\|x-T y\|+\|x-S y\|] \\
& +a_{3}[\|y-T x\|+\|y-S x\|] .
\end{aligned}
$$

Condition (b) gives

$$
\begin{aligned}
&\|T x-T y\|+\|S x-S y\| \\
& \leq a\|x-y\|+\frac{\mu}{r}\|2 x-(T+S) x\|+\frac{\mu}{r}\|2 y-(T+S) y\| \\
&+\frac{a_{2}}{r}\|2 x-(T+S) y\|+\frac{a_{3}}{r}\|2 y-(T+S) x\| \\
& \leq a\|x-y\|+\frac{\mu}{r}\|x-(T+S) x\|+\frac{\mu}{r}\|y-(T+S) y\| \\
&+\frac{a_{2}}{r}\|x-(T+S) y\|+\frac{a_{3}}{r}\|y-(T+S) x\| \\
&+2 \mu+a_{2}+a_{3}, \quad \text { where } a=a_{1}+\alpha .
\end{aligned}
$$

Taking $2 \mu+a_{2}+a_{3}$ sufficiently small, and we conclude that

$$
\begin{gathered}
\|(T+S) x-(T+S) y\| \leq \\
a\|x-y\|+\frac{\mu}{r}\|x-(T+S) x\|+\frac{\mu}{r}\|y-(T+S) y\| \\
+\frac{a_{2}}{r}\|x-(T+S) y\|+\frac{a_{3}}{r}\|y-(T+S) x\| .
\end{gathered}
$$

Thus, $T+S \in H R\left(a, \mu / r, \mu / r, a_{2} / r, a_{3} / r\right), a=a_{1}+\alpha$.

Theorem 2.6 Let $X$ be a Banach space, and $T, S: B_{X}(r) \longrightarrow B_{X}(r)$. Assume the following conditions hold:

(a) $T \in H R\left(a_{1}, a_{2}, a_{3}, a_{4}, a_{5}\right)$.

(b) For each $x \in X$, the Diaz-Metcalf's condition (1) holds.

Then $T+S \in H R\left(a, b / r, c / r, a_{4} / r, a_{5} / r\right)$, if $S \in D(\alpha, \beta), a=\alpha+a_{1}, b=\max \left(a_{2}, \beta\right), c=$ $\max \left(a_{3}, \beta\right)$.

Proof Let $x, y \in B_{X}(r)$,

$$
\begin{gathered}
\|T x-T y\| \leq a_{1}\|x-y\|+a_{2}\|x-T x\|+a_{3}\|y-T y\| \\
+a_{4}\|x-T y\|+a_{5}\|y-T x\|, \\
\|S x-S y\| \leq \alpha\|x-y\|+\mu\left[\|x-S x\|+b_{3}\|y-S y\|\right] ;
\end{gathered}
$$


then

$$
\begin{aligned}
& \|T x-T y\|+\|S x-S y\| \\
& \quad \leq\left(a_{1}+\alpha\right)\|x-y\|+\beta\|x-S x\|+\beta\|y-S y\| \\
& \quad+a_{2}\|x-T x\|+a_{3}\|y-T y\|+a_{4}\|x-T y\|+a_{5}\|y-T x\| ;
\end{aligned}
$$

now, adding the following term to the right hand side of the inequality above:

$$
a_{4}\|x-S y\|+a_{5}\|y-S x\|,
$$

we have

$$
\begin{aligned}
\|T x-T y\|+\|S x-S y\| \leq & \left(a_{1}+\alpha\right)\|x-y\|+\beta\|x-S x\|+\beta\|y-S y\| \\
& +a_{2}\|x-T x\|+a_{3}\|y-T y\|+a_{4}[\|x-T y\| \\
& +\|x-S y\|]+a_{5}[\|y-T x\|+\|y-S x\|] .
\end{aligned}
$$

From the Diaz-Metcalf inequality we conclude

$$
\begin{aligned}
& \|T x-T y\|+\|S x-S y\| \\
& \leq a\|x-y\|+\frac{b}{r}\|2 x-(T+S) x\|+\frac{c}{r}\|2 y-(T+S) y\| \\
& +\frac{a_{4}}{r}\|2 x-(T+S) y\|+\frac{a_{5}}{r}\|2 y-(T+S) x\| \\
& \leq a\|x-y\|+\frac{b}{r}\|x-(T+S) x\|+\frac{c}{r}\|y-(T+S) y\| \\
& +\frac{a_{4}}{r}\|x-(T+S) y\|+\frac{a_{5}}{r}\|y-(T+S) x\|+b+c+a_{4}+a_{5} \\
& \text { where } a=a_{1}+\alpha, b=\max \left(a_{2}, \beta\right), c=\max \left(a_{3}, \beta\right) \text {. }
\end{aligned}
$$

Taking $b+c+a_{4}+a_{5}$ sufficient small, we conclude that

$$
\begin{aligned}
\|(T+S) x-(T+S) y\| \leq & a\|x-y\|+\frac{b}{r}\|x-(T+S) x\|+\frac{c}{r}\|y-(T+S) y\| \\
& +\frac{a_{4}}{r}\|x-(T+S) y\|+\frac{a_{5}}{r}\|y-(T+S) x\| .
\end{aligned}
$$

Hence, $T+S \in H R\left(a, b / r, c / r, a_{4} / r, a_{5} / r\right), a=a_{1}+\alpha, b=\max \left(a_{2}, \beta\right), c=\max \left(a_{3}, \beta\right)$.

\section{Further results}

All the results given in the past section can be rewritten for maps acting on the unit ball $B_{X}(1)$, by taking the contractive parameters on each class conveniently small (smaller than the constant $r$ ). In such a case, the proofs of the previous results run analogously with obvious changes.

Also, we would like to point out that there are several inequalities of the type DiazMetcalf that can replace the condition (b) in our results. These alternatives include the consideration of more than one linear functional, as well as the explicit construction of such a functional for the case of Hilbert spaces; see [19]. 


\subsection{The case of strictly convex Banach spaces}

As we saw, condition (b) in the previous results implies that we can guarantee a reverse triangle inequality which allows us to obtain the conclusions. If we consider strictly convex Banach spaces, as in [18], we can obtain the same kind of conclusions by replacing the condition (b) by a more suitable one.

Definition 1 (see [20]) A norm $\|\cdot\|$ on a Banach space is called strictly convex if whenever $\|x\|=\|y\|=1$ and $\|x+y\|=2$ then necessarily $x=y$.

A Banach space $X$ is said to be strictly convex if its norm is strictly convex.

In these spaces we can assure that $\|x+y\|=\|x\|+\|y\|$ if $x=\lambda y$, for any scalar $\lambda$. So, changing the condition (b) in our results leads to the following:

(b') $x-T x=k(x-S x)$ for any scalar $k$ and every $x \in B_{X}(1)$

and we obtain the same conclusions as before.

Let $A$ and $B$ be classes of mappings. By $A+B$ we will mean the sum of the mappings $T \in A$ and $S \in B$, and $A+B=C$ will mean that the mapping $T+S$ belongs to the class $C$.

Similar conclusions as of Theorems 2.1, 2.2, 2.3, 2.4, 2.5, and 2.6 are obtained for $x$ out of $B_{X}(1)$ and for $y$ any scalar multiple of $x$.

Proposition 3.1 Let $X$ be a strictly convex Banach space, and $T, S: X \longrightarrow X$, suppose that $x-T y=r(x-S y)$ for any scalar $r$ and every $x, y \in X$. Assume in addition, $x=\lambda y$. Then

(i) $B C(\alpha)+K A(\beta)=D(\mu, \beta), \mu=\alpha+\beta$.

(ii) $B C(\alpha)+C H(\beta)=R H(\mu, \beta, \beta), \mu=\alpha+\beta$.

(iii) $B C(\alpha)+R E\left(a_{1}, a_{2}, a_{3}\right)=R E\left(\mu, a_{2}, a_{3}\right), \mu=\alpha+a_{1}+\max \left(a_{2}, a_{3}\right)$.

(iv) $B C(\alpha)+R H\left(a_{1}, a_{2}, a_{3}\right)=R H\left(\mu, a_{2}, a_{3}\right), \mu=\alpha+a_{1}+\max \left(a_{2}, a_{3}\right)$.

(v) $B C(\alpha)+H R\left(a_{1}, a_{2}, a_{3}, a_{4}, a_{5}\right)=H R\left(\mu, a_{2}, a_{3}, a_{4}, a_{5}\right)$, $\mu=\alpha+a_{1}+\max \left(a_{2}+a_{4}, a_{3}+a_{5}\right)$.

(vi) $B C(\alpha)+D(a, b)=D(\mu, b), \mu=\alpha+a+b$.

(vii) $K A(\alpha)+C H(\beta)=H R(\mu, \alpha, \alpha, \beta, \beta), \mu=2(\alpha+\beta)$.

(viii) $K A(\alpha)+R E\left(a_{1}, a_{2}, a_{3}\right)=R E(\mu, a, b), \mu=a_{1}+\max (a, b), a=\max \left(\alpha, a_{2}\right)$, $b=\max \left(\alpha, a_{3}\right)$.

(ix) $K A(\alpha)+R H\left(a_{1}, a_{2}, a_{3}\right)=H R\left(\mu, \alpha, \alpha, a_{2}, a_{3}\right), \mu=a_{1}+\max \left(\alpha+a_{2}, \alpha+a_{3}\right)$.

(x) $K A(\alpha)+H R\left(a_{1}, a_{2}, a_{3}, a_{4}, a_{5}\right)=H R\left(\mu, a, b, a_{4}, a_{5}\right), \mu=a_{1}+\max \left(a+a_{4}, b+a_{5}\right)$, $a=\max \left(\alpha, a_{2}\right), b=\max \left(\alpha, a_{3}\right)$.

(xi) $K A(\alpha)+D(a, b)=D(\beta, \mu), \beta=a+\mu, \mu=\max (\alpha, b)$.

(xii) $C H(\alpha)+R E\left(a_{1}, a_{2}, a_{3}\right)=H R\left(\mu, \alpha, \alpha, a_{2}, a_{3}\right), \mu=a_{1}+\max \left(\alpha+a_{2}, \alpha+a_{3}\right)$.

(xiii) $C H(\alpha)+R H\left(a_{1}, a_{2}, a_{3}\right)=R H(\mu, a, b), \mu=a_{1}+\max (a, b), a=\max \left(\alpha, a_{2}\right)$, $b=\max \left(\alpha, a_{3}\right)$.

(xiv) $C H(\alpha)+H R\left(a_{1}, a_{2}, a_{3}, a_{4}, a_{5}\right)=H R\left(\mu, a_{2}, a_{3}, a, b\right), \mu=a_{1}+\max \left(a_{2}+a, a_{3}+b\right)$, $a=\max \left(\alpha, a_{4}\right), b=\max \left(\alpha, a_{5}\right)$.

(xv) $C H(\alpha)+D(a, b)=H R(\mu, b, b, \alpha, \alpha), \mu=\alpha+a$.

(xvi) $R E\left(a_{1}, a_{2}, a_{3}\right)+R H\left(b_{1}, b_{2}, b_{3}\right)=H R\left(\mu, a_{2}, a_{3}, b_{2}, b_{3}\right)$, $\mu=a_{1}+b_{1}+\max \left(a_{2}+b_{2}, a_{3}+b_{3}\right)$.

(xvii) $R E\left(a_{1}, a_{2}, a_{3}\right)+H R\left(b_{1}, b_{2}, b_{3}, b_{4}, b_{5}\right)=H R\left(\mu, b, c, b_{4}, b_{5}\right)$, $\mu=a_{1}+b_{1}+\max \left(b+b_{4}, c+b_{5}\right), b=\max \left(a_{2}, b_{2}\right), c=\max \left(a_{3}, b_{3}\right)$. 
(xviii) $R E\left(a_{1}, a_{2}, a_{3}\right)+D(a, b)=D(\gamma, \mu), \mu=\max \left(a_{2}, a_{3}, b\right), \gamma=a_{1}+a+\mu$.

(xix) $R H\left(a_{1}, a_{2}, a_{3}\right)+H R\left(b_{1}, b_{2}, b_{3}, b_{4}, b_{5}\right)=H R\left(\mu, b_{2}, b_{3}, b, c\right)$, $\mu=a_{1}+\max \left(b_{2}+b, b_{3}+c\right), b=\max \left(a_{2}, b_{4}\right), c=\max \left(a_{3}, b_{5}\right)$.

(xx) $R H\left(a_{1}, a_{2}, a_{3}\right)+D(a, b)=H R\left(\mu, b, b, a_{2}, a_{3}\right), \mu=a_{1}+a+\max \left(b+a_{2}, b+a_{3}\right)$.

(xxi) $H R\left(a_{1}, a_{2}, a_{3}, a_{4}, a_{5}\right)+D(a, b)=H R\left(\mu, \beta, \gamma, a_{4}, a_{5}\right)$, $\mu=a+a_{1}+\max \left(b+a_{4}, b+a_{5}\right), \beta=\max \left(a_{2}, b\right), \gamma=\max \left(a_{3}, b\right)$.

Proof The proof follows from Theorems 2.1, 2.2, 2.3, 2.4, 2.5, and 2.6. Using the hypothesis $x=\lambda y$, we can guarantee that $\|x\|+\|y\|=\|x-y\|$, the rest is a repeat of each proof of the theorems mentioned above.

Notice that for the parameters $\phi$ in the terms $\|x-y\|($ i.e. $\phi\|x-y\|)$ in the contractive inequalities of Proposition 3.1, some of the combinations with the other respective parameters are not unique. For example, in (viii) we can put $\mu=a_{1}+\max \left(\alpha+a_{2}, \alpha+a_{3}\right)$.

\subsubsection{Examples}

Now, we will give examples showing that the conditions in the results above are necessary.

Example 1 Let $X$ be a Banach space and consider next the map

$$
\begin{aligned}
& T: X \longrightarrow X, \\
& T x=\frac{x}{p}, \quad p \in \mathbb{R} .
\end{aligned}
$$

For $p>1, T \in B C\left(\frac{1}{p}\right)$.

Now, we consider the map

$$
\begin{aligned}
& S: X \longrightarrow X, \\
& S x=\frac{p-1}{p} x, \quad p \in \mathbb{R} .
\end{aligned}
$$

Notice that $S$ satisfies

$$
\|S x-S y\| \leq \beta[\|x-S x\|+\|y-S x\|] \quad \text { for } \beta=p-1
$$

For instance, taking $p=\frac{4}{3}$ we have $T \in B C\left(\frac{3}{4}\right)$ and $S \in K A\left(\frac{1}{3}\right)$.

However, $(T+S) x=x$, and $T+S \notin D\left(\frac{3}{4}, \frac{1}{3}\right)$. So Theorem 2.1 fails because $x-T y \neq r(x-S y)$ for any scalar $r$ and every $x, y \in X$.

Let us note that in this example condition $X$ of a strictly convex Banach space is not sufficient for the conclusion of Theorem 2.1.

Example 2 Let us consider the following maps:

$$
\begin{aligned}
& T:\left(\mathbb{R}^{2},\|\cdot\|_{2}\right) \longrightarrow\left(\mathbb{R}^{2},\|\cdot\|_{2}\right), \\
& T(x, y)=\left(\frac{x}{4}, \frac{y}{4}\right)
\end{aligned}
$$


and

$$
\begin{aligned}
& S:\left(\mathbb{R}^{2},\|\cdot\|_{2}\right) \longrightarrow\left(\mathbb{R}^{2},\|\cdot\|_{2}\right), \\
& S(x, y)=\left(\frac{7}{18} x, \frac{7}{18} y\right) .
\end{aligned}
$$

Here $\|(x, y)\|_{2}=\sqrt{x^{2}+y^{2}}$. It is well known that $\left(\mathbb{R}^{2},\|\cdot\|_{2}\right)$ is a strictly convex Banach space. Then $T$ and $S$ satisfy the following:

(1) $T \in B C\left(\frac{1}{2}\right)$.

(2) $S \in D\left(\frac{5}{72}, \frac{20}{72}\right)$.

(3) $\left(y_{1}, y_{2}\right)-T\left(x_{1}, x_{2}\right)=r\left[\left(y_{1}, y_{2}\right)-S\left(x_{1}, x_{2}\right)\right]$ with $r=9$ and $\left(y_{1}, y_{2}\right)=\frac{1}{8}\left(x_{1}, x_{2}\right)$.

Also, we can check that $S+T \in D\left(\frac{41}{72}, \frac{20}{72}\right)$.

On the other hand, if we take $\left(x_{1}, y_{1}\right)=(3,0)$ and $\left(x_{2}, y_{2}\right)=(0,1)$, some computation shows that Theorem 2.1 (vi) is not satisfied because $(0,1),(3,0) \notin\left(B_{\mathbb{R}^{2}},\|\cdot\|_{2}\right)$. But, for $\left(x_{1}, y_{1}\right)=(2,0)$ and $\left(x_{2}, y_{2}\right)=(4,0)$, by computation we can verify that $T+S \in D\left(\frac{41}{72}, \frac{20}{72}\right)$. Hence Proposition 3.1 is satisfied.

Example 3 For the maps on the Example 2, we might take $\left(\mathbb{R}^{2},\|\cdot\|_{\infty}\right)$ instead of $\left(\mathbb{R}^{2},\|\cdot\|_{2}\right)$, where $\|(x, y)\|_{\infty}=\max (|x|,|y|)$. Then we can see that $T+S \notin D\left(\frac{41}{72}, \frac{20}{72}\right)$. To verify this it is sufficient to take $\left(x_{1}, x_{2}\right)=\left(\frac{1}{2}, 0\right)$ and $\left(x_{2}, y_{2}\right)=(0,0)$. This proves that the condition ' $X$ be a strictly convex Banach space' is necessary. (Because $\left(\mathbb{R}^{2},\|\cdot\|_{\infty}\right)$ is not a strictly convex Banach space.)

\section{Competing interests}

The authors declare that they have no competing interests.

\section{Authors' contributions}

All authors contributed equally in writing this paper, and read and approved the final manuscript.

\section{Author details}

${ }^{1}$ Departamento de Matemáticas, Pontificia Universidad Javeriana, Bogotá, Colombia. ${ }^{2}$ Departamento de Matemáticas, Universidad de Los Andes, Mérida, 5101-A, Venezuela.

\section{Acknowledgements}

The authors are thankful to the referees for very constructive comments and suggestions.

Received: 23 September 2013 Accepted: 7 May 2014 Published: 23 May 2014

\section{References}

1. Morales, JR, Rojas, EM: On the sum of contractive type of mappings I: maps on the same class. In: Mityushev, V, Ruzhansky, M (eds.) Trends in Mathematics/Research Prospectives. Birkhäuser, Basel (to appear)

2. Diaz, JB, Metcalf, FT: A complementary triangle inequality in Hilbert and Banach spaces. Proc. Am. Math. Soc. 17(1), 88-97 (1966)

3. Istrăţescu, V: Fixed Point Theory: An Introduction. Mathematics and Its Applications, vol. 7. Reidel, Boston (1981)

4. Kannan, R: Some results on fixed points II. Am. Math. Mon. 76, 405-408 (1969)

5. Kannan, R: Some results on fixed points III. Fundam. Math. 70, 169-177 (1971)

6. Chatterjea, SK: Fixed point theorem. C. R. Acad. Bulgare Sci. 25, 727-730 (1972)

7. Reich, S: Kannan's fixed point theorems. Boll. Unione Mat. Ital. 4, 121-128 (1971)

8. Reich, S: Remarks concerning contraction mappings. Can. Math. Bull. 14, 121-124 (1971)

9. Reich, S: Remarks on fixed points. Atti Accad. Naz. Lincei, Rend. Cl. Sci. Fis. Mat. Nat. (8) 52, 689-697 (1972)

10. Rhoades, BE: A comparison of various definitions of contractive mappings. Trans. Am. Math. Soc. 226, $257-290$ (1977)

11. Kincses, J, Totik, V: Theorems and counterexamples on contractive mappings. Math. Balk. 4(1), 69-90 (1990)

12. Hardy, GE, Rogers, TD: A generalization of a fixed point theorem of Reich. Can. Math. Bull. 16, $201-206$ (1973)

13. Goebel, K, Kirk, WA, Chimi, TN: A fixed point theorem in uniformly convex spaces. Boll. Unione Mat. Ital. 7, 67-75 (1973)

14. Lopez-Gomez, J: A fixed point theorems for discontinuous operators. Glas. Mat. 23, 115-118 (1988)

15. Nova, L: Fixed point theorems for some discontinuous operators. Pac. J. Math. 123, 189-196 (1986) 
16. Derryck, WR, Nova, L: Interior Properties and Fixed Points of Certain Discontinuous Operators, pp. 239-245. Elsevier, Amsterdam (1992)

17. Derrick, WR, Nova, L: Fixed point theorems for discontinuous operators. Glas. Mat. 24, 339-347 (1989)

18. Morales, R, Rojas, E: Some results for one class of discontinuous operators with fixed points. Acta Math. Univ. Comen. $\operatorname{LXXVI}(2), 149-160$ (2007)

19. Dragomir, SS: Reverses of the triangle inequality in Banach spaces. J. Inequal. Pure Appl. Math. 6(5), 129 (2005)

20. Istrăţescu, V: Strictly Convexity and Complex Strictly Convexity: Theory and Applications. Lecture Notes in Pure and Applied Math., vol. 98. Dekker, New York (1984)

10.1186/1029-242X-2014-208

Cite this article as: Rojas and Morales: On the sum of contractive type of mappings II: maps on different classes. Journal of Inequalities and Applications 2014, 2014:208

Submit your manuscript to a SpringerOpen ${ }^{\circ}$ journal and benefit from:

- Convenient online submission

- Rigorous peer review

- Immediate publication on acceptance

Open access: articles freely available online

- High visibility within the field

- Retaining the copyright to your article

Submit your next manuscript at $>$ springeropen.com 